QFET-2017-18

SI-HEP-2017-19

\title{
Charged lepton flavour violation in a Pati-Salam model with gauged flavour symmetry
}

\author{
Paul Moch* \\ Theoretische Physik 1, Naturwissenschaftlich-Technische Fakultät, Universität Siegen, \\ Walter-Flex-Straße 3, 57072 Siegen, Germany \\ E-mail: paulmoch@physik.uni-siegen.de
}

\begin{abstract}
We describe the computation of charged lepton flavour violating processes such as $\mu \rightarrow e \gamma, \mu \rightarrow$ $3 e$ and Muon conversion in the vicinity of nuclei in a Pati-Salam (PS) model with gauged flavour symmetry. Our model combines PS and flavour symmetries in a renormalisable framework, which allows for realistic masses and mixing angles in the quark and lepton sector. The PS symmetry is broken by vacuum expectation values of scalar fields with non-trivial flavour quantum numbers, which generate different flavour structures for up-type quarks, down-type quarks and leptons. Due to the mixture of new heavy fermions with the Standard Model fermions sizeable, tree-level flavour violating interactions are generated. We apply the current and future limits for charged lepton flavour violating processes to the parameter space of our model.
\end{abstract}

EPS-HEP 2017, European Physical Society conference on High Energy Physics,

5-12 July 2017 Venice, Italy

${ }^{*}$ Speaker. 


\section{Introduction}

The discovery of the Higgs boson at the LHC in 2012 [1,2] finally opens the era of direct tests of the electroweak symmetry breaking mechanism and the Yukawa sector of the Standard Model (SM) together with the underlying question about the origin of quark and lepton masses and mixing.

The explanation for the numerical values of the a priori undetermined SM Yukawas matrices calls for theories beyond the SM (BSM). As an example, we present in this article an explicitly leftright symmetric grand unified theory (GUT) which is based on the PS gauge group [3,4]. The flavour structure is implemented by extending the framework of the Grinstein, Redi and Villadoro (GRV) [5] model.

The PS gauge group allows gauge coupling unification via several intermediate mass scales [6-13]. One of the feature is the incorporation of all SM multiplets( plus the right handed neutrino) into only two PS multiplets. The full symmetry of the Lagrangian is given by

$$
\underbrace{\left(S U(4) \times S U(2) \times S U(2)^{\prime}\right)}_{\text {Pati-Salam }} \times \underbrace{\left(S U(3)_{I} \times S U(3)_{I I}\right)}_{\text {flavour }},
$$

where the $\mathrm{SU}(2)$ is associated to the SM particles, while the new heavy particles are connected to the $\mathrm{SU}(2)^{\prime}$ gauge group. The phenomenology of the quark sector was investigated thoroughly for this setup in [14].

Here we report on a similar analysis done in the lepton sector [15]. This work extended the model to the charged lepton sector, involving additional Pati-Salam and flavour-symmetry breaking flavon fields. It includes an extensive study of the lepton-flavour violating (LFV) phenomenology.

The content of the remaining article is as follows. Section two contains a short description of the extension of the model in [14] to the lepton sector. In addition, the most important flavour changing anomalous tree-level couplings contributing to the charged-lepton sector are presented. In section 3 we consider the phenomenological implications of these anomalous couplings to the LFV observables $\mu \rightarrow e \gamma, \mu \rightarrow 3 e$ and muon conversion in the vicinity of nuclei. We conclude in the last section.

\section{A Pati-Salam model with viable charged lepton sector}

The setup of the left-right symmetric Pati-Salam model with gauged $S U(3)_{I} \times S U(3)_{I I}$ flavour symmetry has been discussed in [14]. The left- and right-chiral Pati-Salam multiplets $q_{L, R}$ contain all SM fermions. Additional fermionic partners $\left(\Sigma_{L, R}, \Xi_{L, R}\right)$ are introduced in order to formulate a renormalisable model in which the flavour symmetry is broken by vacuum expectation values (VEV) of matrix-valued scalar fields $S$ and $T^{\prime}$. The flavon field transforms trivially under PS, while $T^{\prime}$ furnishes a triplet representation of the PS gauge factor $S U(2)^{\prime}$. The combination of flavons in both the singlet and triplet representation of $S U(2)^{\prime}$ is necessary to distinguish up-type from downtype flavour structures. To first approximation, the Yukawa matrices of the light (i.e. SM) fermions 
are obtained by integrating out the heavy partners. More accurately, it is necessary to diagonalize a $9 \times 9$ mass matrix in order to identify the correct mass eigenstates. This is particularly relevant for the up-type sector where the two partners of the top quark are only marginally heavier than the top itself. To cancel gauge anomalies this model introduces PS neutral fermions $\Theta_{L, R}$ which acquire Majorana masses through extra scalars $S_{v}^{(\prime)}$. The coupling of $\Theta_{L}$ to the neutral component of $\Sigma_{R}$ induces a heavy Majorana mass for the latter, which in turn generates light neutrino masses via the seesaw mechanism. We refer the reader to [14] for further details on the construction of the model. The complete particle content of the Pati-Salam model can be found in [14]. It yields a renormalisable Yukawa Lagrangian of the form

$$
\mathscr{L}_{\text {Yuk }}=\mathscr{L}_{\text {Yuk }}^{q}+\mathscr{L}_{\text {Yuk }}^{v}
$$

with

$$
\begin{aligned}
\mathscr{L}_{\text {Yuk }}^{q}= & \lambda \bar{q}_{L} H \Sigma_{R}+\bar{\Sigma}_{L}\left(\kappa_{S} S+\kappa_{T} T^{\prime}\right) \Sigma_{R}+M \bar{\Sigma}_{L} q_{R}+\text { h.c. } \\
& +\lambda \bar{\Xi}_{L} H q_{R}+\bar{\Xi}_{L}\left(\kappa_{S} S+\kappa_{T} T\right) \Xi_{R}+M \bar{q}_{L} \Xi_{R}+\text { h.c. },
\end{aligned}
$$

and

$$
\mathscr{L}_{\text {Yuk }}^{v} \sim \bar{\Theta}_{L} \Phi^{\prime} \Sigma_{R}+\frac{1}{2} \bar{\Theta}_{L} S_{v} \bar{\Theta}_{L}+\bar{\Xi}_{L} \Phi \Theta_{R}+\frac{1}{2} \Theta_{R} S_{V}^{\prime} \Theta_{R}+\bar{\Theta}_{L} S^{\dagger} \Theta_{R}+\text { h.c. . }
$$

The Lagrangian in Eq. (2.2) describes the Yukawa structure of the charged fermions. A comprehensive discussion of the quark sector can be found in [14]. Looking at the charged lepton sector it becomes clear that the effective Yukawa matrix $Y_{\ell}$ is identical to the down-type quark Yukawa matrix $Y_{d}$. Thus to achieve realistic fermion mass patterns one has to modify the model. To this end we extended the scalar sector by additional flavour symmetry breaking flavon fields in the adjoint representation of $S U(4)$. This amounts to simple replacements in Eq. (2.2) such as

$$
\bar{\Sigma}_{L}\left(\kappa_{S} S\right) \Sigma_{R} \rightarrow \bar{\Sigma}_{L}\left(\kappa_{S} S_{1}+\kappa_{S_{15}} S_{15}\right) \Sigma_{R},
$$

which distinguishes the quark and lepton components in the heavy fermions and eventually leads to different effective Yukawa matrix $Y_{\ell}$ from $Y_{d}$.

Following the notations of [14] we can then write down the approximate effective Yukawa matrix of the charged leptons as

$$
Y_{\ell} \simeq-\lambda\left[\frac{1}{s_{l}-t_{l}^{\prime}}+\frac{1}{s_{l}}\right]
$$

where $s_{i}=\kappa_{S_{i}}\left\langle S_{i}\right\rangle / M$ and $t_{i}=\kappa_{T_{i}}\left\langle T_{i}\right\rangle / M$ are dimensionless $3 \times 3$ matrices. Compared to the quark case [14], the relations $s_{l}, t_{l}^{\prime} \gg 1$ are generally satisfied for the leptons [15]. Therefore Eq. (2.5) provides a good approximation even for the third generation and can be used as a starting point to constrain of the free parameters.

We note that the free parameters of the theory are the matrices $s_{l}$ and $t_{l}^{\prime}$ in the lepton sector. Therefore in order to generate the correct lepton masses one can replace $s_{l}-t_{l}^{\prime}$ in favour of $Y_{\ell}$ using 
Eq. (2.5). To this end we use a basis in which $Y_{\ell}$ is diagonal to find the relation

$$
s_{l}-t_{l}^{\prime} \rightarrow-\left[\frac{\hat{Y}_{\ell}}{\lambda}+s_{l}^{-1}\right]^{-1}=-\left[\frac{\sqrt{2} \hat{M}^{\ell}}{\lambda v_{d}}+s_{l}^{-1}\right]^{-1},
$$

where hats denote diagonal $3 \times 3$ matrices, $\hat{M}^{\ell}=\operatorname{Diag}\left(m_{e}, m_{\mu}, m_{\tau}\right)$ contains the measured chargedlepton masses and $v_{d}=v / \sqrt{1+\tan ^{2} \beta}$. With the elimination of $t_{l}^{\prime}$ the charged-lepton masses are automatically correctly reproduced, independent of the flavour structure encoded in $s_{l}$ [15].

The LFV contribution to the charged lepton sector can be parametrised by the Lagrangian

$$
\begin{aligned}
\mathscr{L}_{\mathrm{LFV}}= & \frac{g Z^{\mu}}{2 c_{W}}\left(\Delta g_{Z \bar{\ell}_{L} \ell_{L}}^{i j}\left(\bar{\ell}_{i} \gamma_{\mu} P_{L} \ell_{j}\right)-\Delta g_{Z \bar{\ell}_{R} \ell_{R}}^{i j}\left(\bar{\ell}_{i} \gamma_{\mu} P_{R} \ell_{j}\right)\right) \\
& -\frac{g W^{\mu}}{\sqrt{2}} \Delta g_{W \bar{v}_{L} \ell_{L}}^{i j}\left(\bar{v}_{i} \gamma_{\mu} P_{L} \ell_{j}\right)+\frac{h}{\sqrt{2}} \Delta g_{h \bar{\ell} \ell}^{i j}\left(\bar{\ell}_{i} P_{R} \ell_{j}\right)+\text { h.c. },
\end{aligned}
$$

where the $3 \times 3$ coupling matrices $\Delta g_{Z \bar{\ell}_{L} \ell_{L}}, \Delta g_{Z \bar{\ell}_{R} \ell_{R}}, \Delta g_{W \bar{v}_{L} \ell_{L}}$ are generated by the rotation of the kinetic terms of the Lagrangian into the mass basis $[14,15]$. Tree-level exchange diagrams with the heavy fermion partners contribute to the anomalous coupling to the Higgs $\Delta g_{h \bar{\ell} \ell}$ in this model $[14,15]$. The full analytic expressions in terms of the matrix $s_{l}$ of all above noted anomalous couplings can be found in [15]. A priori this model induces couplings of the SM fermions to the new gauge bosons generated by the $\mathrm{SU}(2)^{\prime}$ gauge group. This leads naturally to dipole and four fermion contributions to the LFV sector. However due to the choice of symmetry breaking [14] the associated gauge bosons are too heavy and therefore these interactions are not relevant for the further analysis.

\section{Numerical Analysis}

In the end, branching ratios of LFV processes under consideration can be boiled down to complicated functions of the free model parameters in the matrix $s_{l}$. Because there are not many restriction for the entries of this matrix we perform a numerical scan over a large portion of the parameter space. In total we employed two different scan strategies. The first one adopted the scan used in [14], i.e.

$$
\lambda \in[1.5,3], \quad \tan \beta \in[1,15], \quad M \in[750,2500] \mathrm{GeV},
$$

where the parameter $M$ sets essentially the mass scale of the new particles. To be more precise, the heavy partner particle of the $\tau$ is expected to have a mass of the order of $\sim M$.

The other scan fixes the scale $M$ at $1 \mathrm{TeV}$ and includes in total two datasets. The first set sets no restrictions on the matrix $s_{l}$ (indicated by blue or dark grey points). The second dataset restricts the mixing angles in the range $[0, \pi / 6]$ for the matrix $s_{l}$. This allows us to investigate the dependence of the various LFV observables on the size of mixing angles.

Our results of the parameter scan are shown in two-dimensional scatter plots. These plots show 


\begin{tabular}{|c|c|c|}
\hline Process & Current limit & Future limit \\
\hline $\operatorname{Br}(\mu \rightarrow e \gamma)$ & $4.2 \times 10^{-13}[16]$ & $6 \times 10^{-14}[17]$ \\
\hline $\operatorname{Br}(\mu \rightarrow 3 e)$ & $1 \times 10^{-12}[18]$ & $1 \times 10^{-16}[19]$ \\
\hline $\operatorname{Br}^{\mathrm{Au}}(\mu N \rightarrow e N)$ & $7 \times 10^{-13}[20]$ & $1 \times 10^{-16}[21]$ \\
\hline
\end{tabular}

Table 1: Current and future experimental limits on the LFV branching ratios.
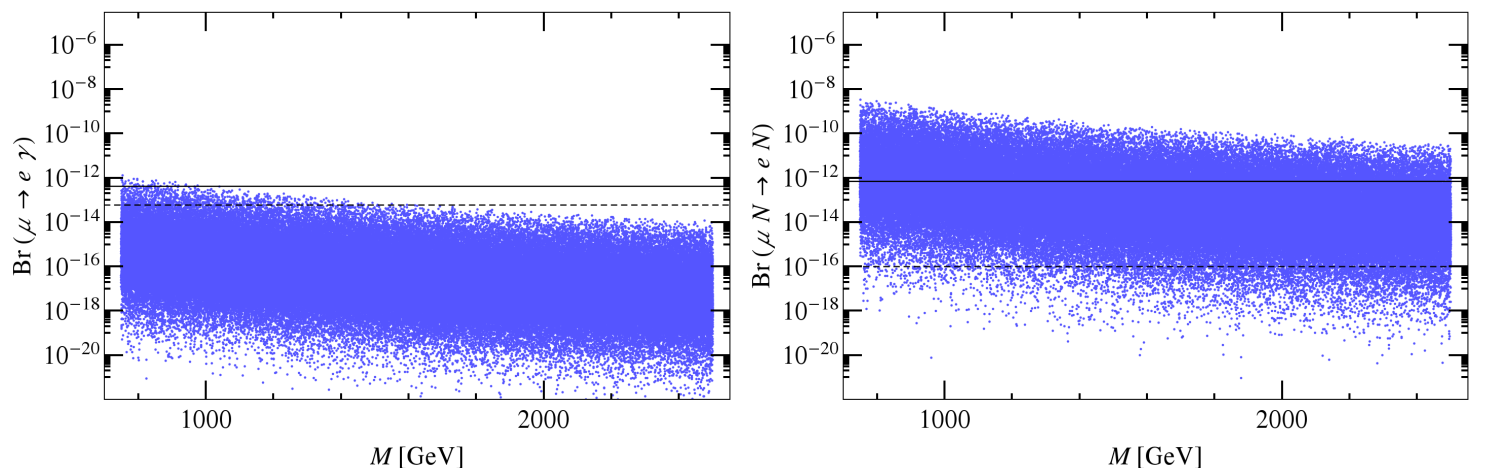

Figure 1: Scatter plots of the branching ratios for $\mu \rightarrow e \gamma$ (left) and $\mu \rightarrow e$ conversion (right) as a function of the NP scale $M$. The solid (dashed) lines indicate the current (future) experimental limits.

the typical range of the branching ratios and the correlation between the observables. We point out that the density of the points does not reflect a probability distribution in the considered model.

Fig. 1 displays the results of the branching ratios $\mu \rightarrow e \gamma$ and the muon conversion in the vicinity of nuclei. Within the chosen range of the NP scale $M$ we observe for the decay $\mu \rightarrow e \gamma$ that the points of the scatter plot are largely compatible with the current and projected future bounds [17]. This can be explained by the absence of tree-level Wilson coefficients contributing to this process. In comparison the muon conversion and the decay $\mu \rightarrow 3 e[15]$ are generated by tree-level couplings, which are given by certain combinations of the anomalous gauge couplings [15]. Therefore the current and future upper limits impose stronger constraints on the parameter space of our model. We point out that our model includes muon conversion rates within the reach of the future experimental bounds. Hence our model predicts possible future LFV signals for this process.

In order to investigate the specific role of the mixing angles in the matrix $s_{l}$ and also the correlations between the different LFV branching ratios, we display in Fig. 2 the correlations between $\mu \rightarrow e \gamma$ and $\mu N \rightarrow e N$, as well as $\mu \rightarrow 3 e$ and $\mu N \rightarrow e N$ for a fixed NP scale, $M=1 \mathrm{TeV}$. Those two plots show that the theory points with reduced mixing angle are restricted to a smaller range of possible branching ratios. The explanation behind this feature is the simple increase of the anomalous couplings with larger mixing angles. $\mu \rightarrow 3 e$ and $\mu N \rightarrow e N$ are generated by a similar set of tree level operators, therefore both processes are strongly correlated. Compared to that the loopinduced decay $\mu \rightarrow e \gamma$ shows only weak correlations $\mu \rightarrow 3 e$ and $\mu N \rightarrow e N$ [15].

The decays of the tau lepton allow another possibility to find LFV signals. However the model 

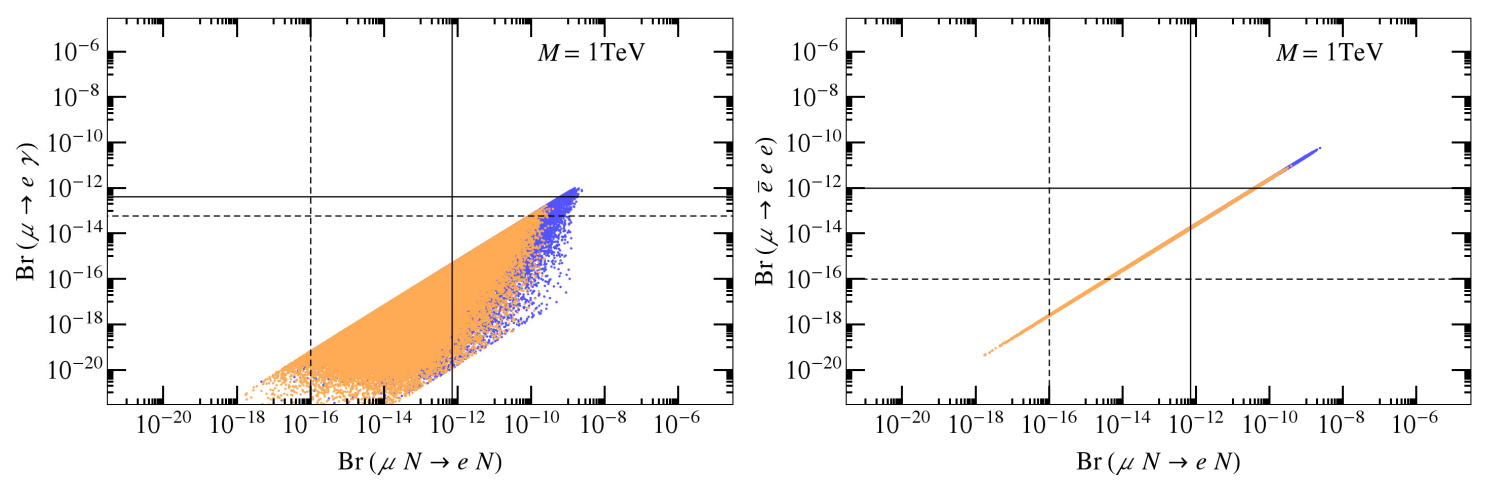

Figure 2: Correlation between the branching ratios for $\mu \rightarrow e \gamma$ vs. $\mu N \rightarrow e N$ (left) as well as $\mu \rightarrow 3 e$ vs. $\mu N \rightarrow e N$ (right). Each plot shows the results for a fixed NP scale, $M=1 \mathrm{TeV}$. The scenario with arbitrary mixing angles is displayed with blue (dark grey) points, while the scenario with mixing angles smaller than $\pi / 6$ is shown with orange (light grey) points.

prediction for branching ratios such as $\tau \rightarrow 3 \mu$ are at least three magnitudes smaller than the best upper limits and will not be accessible in the near future [15].

To conclude the lepton-flavour phenomenology outline of our model we remark shortly on the impact of the electron electric dipole moment (EDM) to the available parameter space. Up to or$\operatorname{der} v_{d}^{2} / M^{2}$ the anomalous couplings of our model are real [15] and therefore do not contribute the electron EDM. As this eliminates dimension-six contributions we estimated the dimensioneight contribution by calculating a corresponding diagram with non-vanishing imaginary parts. We found that this diagram yields electron EDMs with numerical values which are at least two order of magnitudes smaller than the current bounds [22]

$$
\left|d_{e}\right|<8.7 \cdot 10^{-29} e \mathrm{~cm} .
$$

By assuming standard power counting we therefore conclude that the complete dimension-eight contribution to the electron EDM will be suppressed in the same way in our model.

\subsection{Summary}

We presented an extension of a fully renormalisable PS model with gauged flavour $S U(3)_{I} \times$ $S U(3)_{I I}$ to the charged-lepton sector. The model features a realistic generation of the lepton mass matrices. This involves two heavy fermion partners for each SM fermion, whose mixing introduce anomalous SM gauge and Higgs boson couplings. These couplings generate contributions to charged LFV processes, which have been calculated analytically and numerically in [15].

Our numerical scan over a wide range of the possible parameter space of the model indicates that the decay $\mu \rightarrow e \gamma$ may not be seen in the near future. The processes mediated via tree level diagrams with anomalous gauge and Higgs boson couplings such as $\mu \rightarrow 3 e$ decays and $\mu \rightarrow e$ conversion in nuclei show a different picture. Assuming the new-physics scale associated to the heavy fermions in our model is of the order of a few $\mathrm{TeV}$ these two processes are accessible to 
future experiments.

We found that the branching ratios predicted for lepton-flavour violating $\tau$ decays prove to be at least two magnitudes smaller than the corresponding experimental limits. In addition contributions to the electron EDM are suppressed and below the present experimental bounds.

In conclusion, our particular model setup combines the idea of grand unification in the gauge sector and flavour symmetry breaking transferred by new heavy vector-like fermions leading to small flavour-violating effects in the charged-lepton sector.

\section{Acknowledgements}

This work is supported by the Deutsche Forschungsgemeinschaft (DFG) within the Research Unit FOR 1873 (“Quark Flavour Physics and Effective Field Theories”).

\section{References}

[1] G. Aad et al. [ATLAS Collaboration], Phys. Lett. B 716 (2012) 1 [arXiv:1207.7214].

[2] S. Chatrchyan et al. [CMS Collaboration], Phys. Lett. B 716 (2012) 30 [arXiv:1207.7235].

[3] J. C. Pati and A. Salam, Phys. Rev. D 8 (1973) 1240.

[4] J. C. Pati and A. Salam, Phys. Rev. D 10 (1974) 275 [Phys. Rev. D 11 (1975) 703].

[5] B. Grinstein, M. Redi and G. Villadoro, JHEP 1011 (2010) 067 [arXiv:1009.2049].

[6] W. Kilian and J. Reuter, Phys. Lett. B 642 (2006) 81 [hep-ph/0606277].

[7] R. Howl and S. F. King, Phys. Lett. B 652 (2007) 331 [arXiv:0705.0301].

[8] L. Calibbi, L. Ferretti, A. Romanino and R. Ziegler, Phys. Lett. B 672 (2009) 152 [arXiv:0812.0342].

[9] F. Braam, J. Reuter and D. Wiesler, AIP Conf. Proc. 1200 (2010) 458 [arXiv:0909.3081].

[10] V. De Romeri, M. Hirsch and M. Malinsky, Phys. Rev. D 84 (2011) 053012 [arXiv:1107.3412].

[11] C. Arbelaez, R. M. Fonseca, M. Hirsch and J. C. Romão, Phys. Rev. D 87 (2013) 075010 [arXiv:1301.6085].

[12] C. Arbelaez, M. Hirsch, M. Malinsky and J. C. Romão, Phys. Rev. D 89 (2014) 035002 [arXiv:1311.3228].

[13] F. Hartmann, W. Kilian and K. Schnitter, JHEP 1405 (2014) 064 [arXiv:1401.7891].

[14] T. Feldmann, F. Hartmann, W. Kilian and C. Luhn, JHEP 1510 (2015) 160 [arXiv:1506.00782].

[15] T. Feldmann, C. Luhn and P. Moch, JHEP 1611 (2016) 078 [arXiv:1608.04124].

[16] A. M. Baldini et al. [MEG Collaboration], [arXiv:1605.05081].

[17] A. M. Baldini et al., [arXiv:1301.7225].

[18] U. Bellgardt et al. [SINDRUM Collaboration], Nucl. Phys. B 299 (1988) 1.

[19] N. Berger [Mu3e Collaboration], Nucl. Phys. Proc. Suppl. 248-250 (2014) 35. 
[20] W. H. Bertl et al. [SINDRUM II Collaboration], Eur. Phys. J. C 47 (2006) 337.

[21] R. M. Carey et al. [Mu2e Collaboration], FERMILAB-PROPOSAL-0973.

[22] J. Baron et al. [ACME Collaboration], Science 343 (2014) 269 [arXiv:1310.7534 ]. 